\title{
Serial Brain Positron Emission Tomography Fused to Magnetic Resonance Imaging in Post-Infectious and Autoantibody-Associated Autoimmune Encephalitis
}

\author{
David S. Younger ${ }^{1,2}$ \\ ${ }^{1}$ Department of Clinical Medicine, CUNY School of Medicine at City College, New York, NY, USA \\ ${ }^{2}$ The Graduate School of Public Health and Health Policy, City University of New York, New York, NY, USA \\ Email: youngd01@nyu.edu
}

How to cite this paper: Younger, D.S. (2019) Serial Brain Positron Emission Tomography Fused to Magnetic Resonance Imaging in Post-Infectious and Autoantibody-Associated Autoimmune Encephalitis. World Journal of Neuroscience, 9, 153-156.

https://doi.org/10.4236/wjns.2019.93010

Received: July 19, 2019

Accepted: August 3, 2019

Published: August 6, 2019

Copyright (๑) 2019 by author(s) and Scientific Research Publishing Inc. This work is licensed under the Creative Commons Attribution International License (CC BY 4.0).

http://creativecommons.org/licenses/by/4.0/

\begin{abstract}
Serial positron emission tomography fused to magnetic resonance imaging showed progression of GAD65 autoimmune encephalitis.

\section{Keywords}

GAD65, Post-Treatment Lyme disease, Autoimmune Encephalitis, Positron Emission Tomography, Magnetic Resonance Imaging
\end{abstract}

\section{Introduction}

${ }^{18}$ Fluoro-deoxyglucose positron emission tomography fused to magnetic resonance imaging (PET/MRI) with 3-D post-processing for volumetric analysis has been used to confirm the initial diagnosis of seropositive autoimmune encephalitis (AE) [1]. PET has also been used to probe cerebral microglial activation in subjects with post-treatment Lyme disease syndrome (PTLDS) and its link to neurocognitive symptoms [2]. This paper describes progressive cortical hypometabolism seen on serial PET/MRI that paralleled clinical worsening in a patient with PTLDS and emergent AE in association with circulating glutamic acid decarboxylase (GAD) 65 antibodies.

\section{Patient Report}

At age 14, this presently 24-year-old female manifested fatigue, fever, joint pain, dizziness and headaches following an influenza-like illness. She was later diagnosed with Lyme disease and postural orthostatic tachycardia syndrome (POTS) 
and treated with appropriate antibiotics and beta-blockers. Afterward, she described cognitive impairment, recurrent fatigue, sleep disturbance, migraine headaches, depressed mood and obsessive-compulsiveness. At age 19, she sustained one of several concussive head injuries resulting from her first seizure. She later reported insidious worsening of cognitive and neuropsychological worsening associated with several episodes of attenuated psychotic symptoms. Neurological examination showed mild distal sensory loss, weakness, incoordination, tandem gait imbalance, Romberg sign, and mildly impaired memory registration, with grossly intact cranial nerves. In 2017, brain PET/MRI (Figure 1A) showed hypometabolism in the parietal lobes corresponding to areas of volume loss, with hippocampal volumes at the lower limit of normal for age. Low titers of the GAD65 antibody were present in the serum $(1.06 \mathrm{nmol} / \mathrm{L}$; normal $\leq$ 0.02; Mayo Laboratory, MN). Serum Lyme enzyme-linked immunosorbent assay (ELISA) was reactive $(1.15$; normal $<0.91)$ with negative IgG and IgM Lyme Western blot (WB) (New York University Langone Medical Center, NY). She was placed on 2 grams per kilogram monthly intravenous immune globulin (IVIg) therapy however, cognitive and neuropsychological deficits progressed. Brain PET/MRI in 2019 (Figure 1B) showed new areas of hypometabolism in bilateral temporal lobes and cerebellar hemispheres. Temporal lobe volumes were estimated to be at the $10^{\text {th }}$ percentile compared to age-matched controls. Mildly traumatic CSF showed a protein content of $224 \mathrm{mg} / \mathrm{dL}$, 39 nucleated cells, and IgG index 1.28 (normal < 0.66) without GAD65 antibody or oligoclonal bands. Serum and CSF pair (Stony Brook University Lyme Laboratory, NY) showed reactive Lyme serology in serum and CSF pair, with negative IgG and IgM WB. The Lyme index (ratio of CSF/serum-specific antibodies) was 0.86 (normal $<1.1$ ) indicating the absence of active intrathecal infection. She experienced three unprovoked seizures in the weeks following lumbar puncture that were treated with levetiracetam.

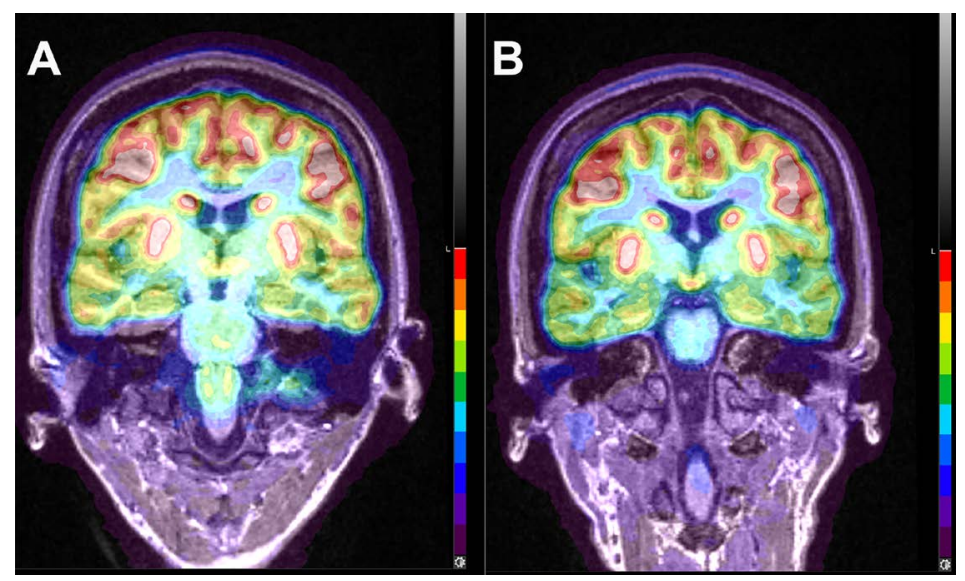

Figure 1. Coronal images of the frontal and temporal lobes of the patient in 2017 (A) and 2019 (B) using blended image of FDG (color-scale) and background volumetric T1-weighted MRI scan. In 2017, there was mild symmetric temporal lobe cortical FDG hypometabolism compared to the frontal cortex that subsequently progressed to more severe FDG hypometabolism in 2019. 


\section{Comment}

Hypometabolism in multiple cortical areas regions is a common early finding in brain PET imaging in cases of AE [3], likely reflecting impaired neuronal activity [4]. The progression of hypometabolism involving the entirety of the temporal lobes in the present patient indicated that treatment with high-dose IVIg alone was not effective in halting progression of the underlying disease process postulated to be post-infectious and autoantibody-associated AE. Autoimmunity targeting the 65 kilo Dalton $(\mathrm{kDa})$ isoform of GAD65 results in diverse disorders from autoimmune diabetes and thyroiditis at low antibody titers $(<2 \mathrm{nmol} / \mathrm{L})$ to temporal lobe epilepsy and $\mathrm{AE}$ at higher titers $(>200 \mathrm{nmol} / \mathrm{L})$ [5]. The neuropathology of diverse forms of $\mathrm{AE}$ is directed at surface and intraneuronal antigens with invasion of the hippocampus by $\mathrm{CD} 8^{+} \mathrm{T}$-cells that express granzyme $\mathrm{B}$ in the presence of main histocompatibility class I molecules present in surviving neurons [6]. Over time, temporal lobe/hippocampal atrophy emerges in areas of encephalitis. Brain PET imaging in patients with PTLDS [2] shows increased expression of the $18 \mathrm{kDa}$ transloctor mitochondrial protein expressed by activated cerebral microglia and reactive astrocytes in widespread cortical areas including the hippocampus, compared to healthy controls, suggesting a relation to encephalopathy. Brain PET/MRI alone does not adequately characterize patients with PTLDS or AE. These respectively necessitate analysis of paired CSF and serum for the detection of Borrelia burgdorferi-specific antibodies to exclude active infection, and assessment of AE-associated autoantibodies in commercially-available panels to search for a causative autoantibody.

While this patient meets criteria for possible AE under recently proposed clinical guidelines that do not depend on neuronal autoantibody status [7], the appropriate management remains uncertain given concomitant PTLDS. The contribution of the low GAD65 antibody to the development of AE is also not well understood. A draft proposal of 2019 Clinical Practice Guidelines of the Infectious Disease Society of America, American Academic of Neurology, and the American College of Rheumatology does not offer guidance in the management of persistent symptoms of Lyme disease after standard of care antibiotic therapy has been administered (unpublished). Nor does it address the relevance of specific findings on brain PET/MRI to PTLDS. Treating physicians faced with similar patients must decide among available therapeutic modalities, including the appropriate use of antibiotics, immune modulatory (IVIg) and biological therapy employing rituximab, to address both post-infectious and neuronal autoantibody-associated immune mechanisms that contribute to cognitive and neuropsychological symptoms associated with progressive worsening cortical hypometabolism.

\section{Acknowledgements}

The author extends appreciation to Timothy Shepherd MD Ph.D., Assistant Professor of Radiology in the Division of Neuroradiology, New York University 
Langone Medical Center, New York, for providing the images and legend for Figure 1; and the patient and her family for permission to publish the case summary.

\section{Conflicts of Interest}

The authors declare no conflicts of interest regarding the publication of this paper.

\section{References}

[1] Vacchiano, V., Giannoccaro, M.P., Napolitano, R.P., Liguori, R., Allegri, V., Rinaldi, R., et al. (2019) Combined Brain Positron Emission Tomography/Magnetic Resonance Imaging in $\mathrm{GABA}_{\mathrm{A}}$ Receptor Encephalitis. European Journal of Neurology, 26. https://doi.org/10.1111/ene.14004

[2] Coughlin, J.M., Yang, T., Rebman, A.W., Kortte, K.B., Du, Y., Mathews, W.B., et al. (2018) Imaging Glial Activation in Patients with Post-Treatment Lyme Disease Symptoms: A Pilot Study Using $\left[{ }^{11} \mathrm{C}\right] \mathrm{DPA}-713$ PET. Journal of Neuroinflammation, 15, 346. https://doi.org/10.1186/s12974-018-1381-4

[3] Probasco, J.C., Solnes, L., Nalluri, A., Cohen, J., Jones, K.M., Zan, E., et al. (2017) Abnormal Brain Metabolism on FDG-PET/CT Is a Common Early Finding in Autoimmune Encephalitis. Neuroimmunology \& Neuroinflammation, 4, e352. https://doi.org/10.1212/NXI.0000000000000352

[4] Dalmau, J., Lancaster, E., Martinez-Hernandez, E., Rosenfeld, M.R. and Balice-Gordon, R. (2011) Clinical Experience and Laboratory Investigations in $\mathrm{Pa}$ tients with Anti-NMDAR Encephalitis. The Lancet Neurology, 10, 63-74. https://doi.org/10.1016/S1474-4422(10)70253-2

[5] McKeon, A. and Tracy, J.A. (2017) GAD65 Neurological Autoimmunity. Muscle \& Nerve, 56, 15-27. https://doi.org/10.1002/mus.25565

[6] Bien, C.G., Vincent, A., Barnett, M.H., Becker, A.J., Blümcke, I., Graus, F., et al. (2012) Immunopathology of Autoantibody-Associated Encephalitides: Clues for Pathogenesis. Brain, 135, 1622-1638. https://doi.org/10.1093/brain/aws082

[7] Graus, F., Titulaer, M.J., Balu, R., Benseler, S., Bien C.G., et al. (2016). A Clinical Approach to Diagnosis of Autoimmune Encephalitis. The Lancet Neurology, 15, 391-404. https://www.ncbi.nlm.nih.gov/pmc/articles/PMC5066574/ 